\title{
RESTORATION OF A POST-SURGICAL DEFECT BY MAGNETIC MAXILLOFACIAL PROSTHESIS: A CASE REPORT
}

\author{
Muhammad Aamir Rafique ${ }^{\mathrm{a}}$, Safana Khan ${ }^{\mathrm{b}}$, Hira Ahmed \\ ${ }^{a}$ Assistant Professor, Department of Prosthodontics, Dental Section Faisalabad Medical University, \\ Faisalabad.

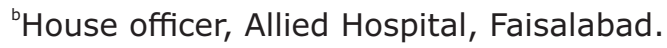 \\ 'Demonstrator, Dental section, Faisalabad Medical University, Faisalabad.
}

\begin{abstract}
:
Edentulous patient with an intra-oral and extra-oral defect suffers from both functional loss and psychological distress. Magnets give a successful approach to rehabilitate the oral functions and improve the quality of life. This case report describes a simple method of fabricating a two part magnetic maxillofacial prosthesis.

KEYWORDS: Maxillofacial Prosthesis, Magnet retained prosthesis, Patient satisfaction, Maggots, Emimaxillectomy, Extra-oral prosthesis, Intra-oral prosthesis.
\end{abstract}

How to cite this:

doi: https://doi.org/10.37723/jumdc.v11i1.350

Rafique MA, Khan S, Ahmed H. RESTORATION OF A POST-SURGICAL DEFECT BY MAGNETIC

MAXILLOFACIAL PROSTHESIS: A CASE REPORT. jumdc. 2020;11(1):44-48.

doi: https://doi.org/10.37723/jumdc.v11i1.350

This is an Open Access article distributed under the terms of the Creative Commons Attribution License (http://creativecommons.org/licenses/by/4.0), which permits unrestricted use, distribution, and reproduction in any medium, provided the original work is properly cited.

\section{INTRODUCTION:}

The surgical treatment of Maxillofacial Malignancies often results in intra-oral and extra-oral defects ${ }^{[1]}$.Intra-oral defects could be of mandible, maxilla, tongue, hard palate, soft palate or cheek. Extra-oral defects constitute both facial and other defects as limbs, digits etc. Facial defects involve auricular, nasal, orbital and ocular defects ${ }^{[2]}$.

Maxillofacial defects involving hard and soft tissues result in communication between oral and nasal cavities. Which cause problems related to esthetic, phonetic and function. Moreover patient's social life is disturbed to a great extend.

These defects can be restored by surgical or non-surgical interventions. Surgical approach involves complex surgical steps. Non-surgical approach for intra-oral defects includes the use of obturator which could be of acrylic or cast. Acrylic obturator is mostly selected for obturator cases having poor socio-economic status; moreover it could easily be repaired and reused as compared to cast. The common problems associated with cast obturator are loss of retention in due course of time because of the plastic deformation ${ }^{[3]}$. Whereas nonsurgical approach for extra-oral prosthesis include prosthesis made up of acrylic or silicone. Silicone prosthesis gives excellent results but is not cost effective as compared to acrylic.

The biggest challenge in Obturator (intra-

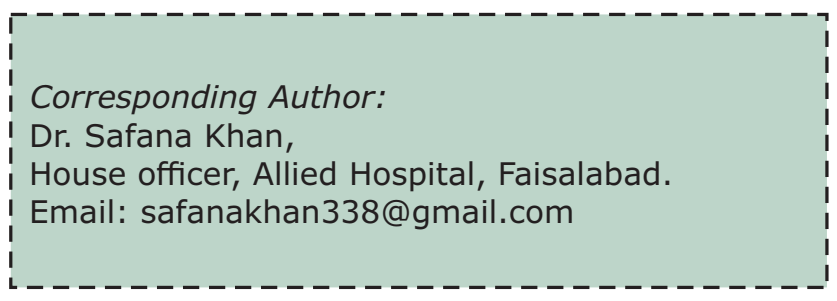


oral/extra-oral) is retention. This can be addressed by various attachment systems i.e. implant retained, adhesive (tissue) retained or magnet retained. Implant restorations can be attached to implant fixture with screw (screwretained) or it can be cemented to an abutment which is attached to implant fixture (cement retained) ${ }^{[4]}$. Magnet retained prosthesis offer retention and it is wear by patients confidently ${ }^{[5]}$. It is smaller in size, has strong attractive forces, easy placement is possible. The majority of prostheses with magnets is sectioned and has a magnet in each section. When the sections are assembled properly, the magnets are attracted to each other and retain the sections ${ }^{[6]}$.

Obturators and facial prosthesis are important not only in rehabilitation of esthetics, phonetics and function but also in patient re-socialization ${ }^{[7]}$. We opted the non-surgical way to rehabilitate the patient as the patient had gone through a major surgery already. By keeping in view the merits and demerits of the attachment systems, we selected the magnetic retained prosthesis.

\section{CASE REPORT:}

A 65 year old edentulous female patient presented to outdoor of Prosthodontics Department of Allied Hospital, Faisalabad Medical University, Faisalabad, with maxillofacial defect (Armani type 2) for a postsurgery prosthetic rehabilitation. She had a history of diabetes mellitus type 2 . She suffered from mucormycosis and maggots were present in the Maxillary sinus. The Anterior and the medial wall of maxillary sinus were necrosed. Partial maxillectomy was done in September 2018 as a treatment modality which also removed part of her left lower cheek.

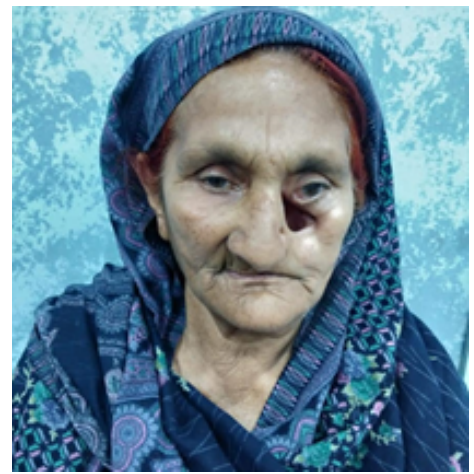

Pre-Operative View

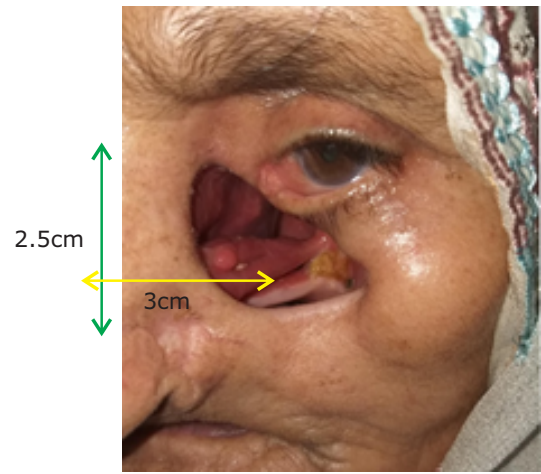

Extra Oral Defect

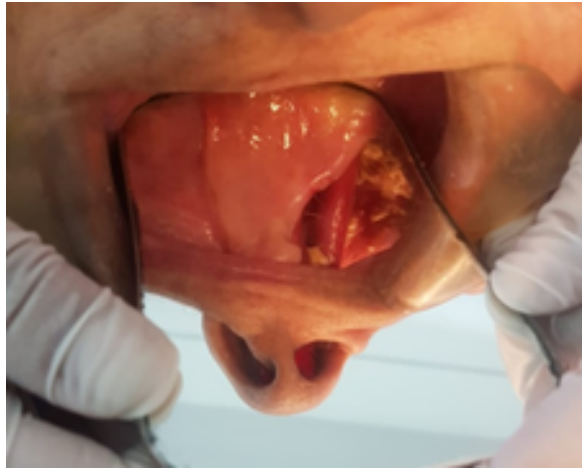

Open communication
Clinical examination revealed open communication between oral and nasal cavities. The patient was informed about different options to manage the defects. Due to her poor socioeconomic status, she chose the nonsurgical procedure. Informed written consent was taken from the patient. The proposed treatment plan was to fabricate a two-section prosthesis fabricated in heat cure acrylic resin which will be held together by incorporation of magnet in between these two sections. Heat cure acrylic resin is a biocompatible material and is used widely for removable prosthesis for both intra and extra oral purposes.

\section{TECHNIQUE:}

The prosthesis was made in two steps i.e. intraoral prosthesis and extra-oral prosthesis. This prosthesis was joined with magnets. The impression was also taken in two steps.

\section{STEP 1:}

\section{FOR INTRA-ORAL PROSTHESIS}

The patient was seated in dental chair in reclined position and drapes placed.

1. An impression of maxillary and mandibular arch was taken using rubber based silicone material (putty material)*(Figure-I). 
2. The impression was poured with dental stone** and casts were prepared.

3. Self-cure trays were prepared and wax rims formed following complete denture instructions ${ }^{[7]}$.

4. Vertical, horizontal and orientation relations were recorded using face bow and transferred to articulator as per guidelines $[8,9]$.

5. After articulation, the tooth setup was done. Bilateral balanced occlusion was maintained.

6. Trial fit was checked for esthetics, retention and occlusion. Prosthesis was cured.

7. After finishing and polishing, maxillary obturator and removable mandibular denture were delivered to patient (Figurev).

\section{STEP 2:}

\section{FOR EXTRA-ORAL PROSTHESIS}

8. Separating media*** was applied to facial hair including eyebrows, eyelids and hair on the forehead to avoid direct contact with the impression material.

9. Eyes were closed using paper tape.

10. Straws were placed in patient's nose to maintain airway during impression.

11. A confiner was created around her face using modeling wax to limit the flow of impression material****. The openings around wax periphery were closed with gauze pieces (Figure-II).

12. The patient was informed about the procedure and she was asked to breathe from nose. She was informed that she will feel cold initially when alginate will touch her face but will become normal in a minute and the final impression will be slightly heavy because of weight of alginate and plaster of Paris. The patient was asked to indicate by raising her hand if she felt any discomfort or difficulty in breathing.

13. Patient was placed to supine position for impression taking.

14. Alginate was mixed with water and poured on patients face. It was ensured that the whole layer of alginate is flowed properly.

15. Plaster of Paris was applied over it to give strength and stability to impression (Figure-III).
16. After the plaster of Paris was set, impression material along with confiner was removed using wangling motion and model poured in dental stone to form master cast (Figure 4). The defect area was duplicated from master cast to form working cast.

17. Wax pattern was made in the form of a cup and a lid. After trial, heat curing was done.

18. Magnets were fixed to the inferior part of the facial prosthesis (cup) and to the most superior part of palatal obturator denture.

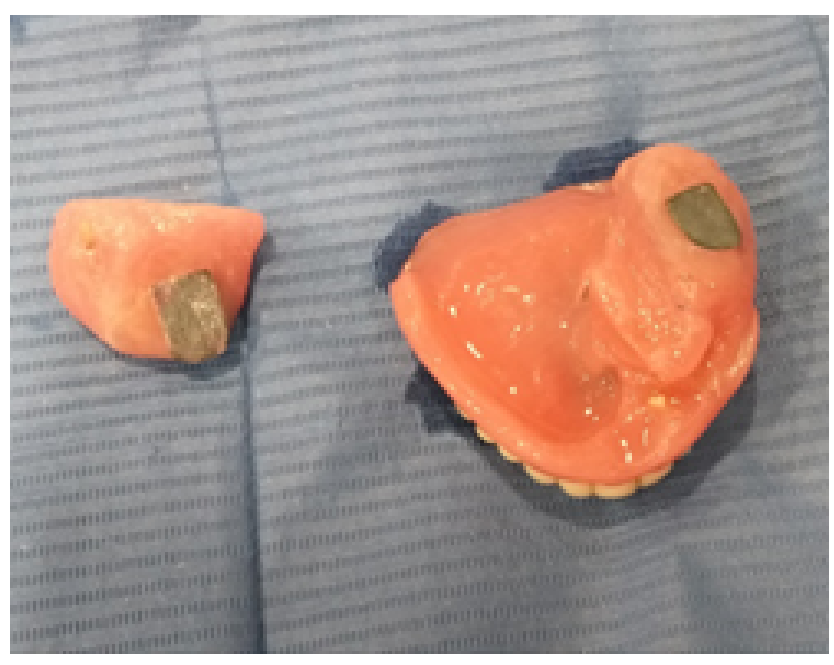

19. Trial fit was performed to check the functional and esthetical requirement.

20. Color techniques were used. Acrylic paints were used for extrinsic and intrinsic staining. It was made sure that the extraoral prosthesis matches with the patient's skin tone. Color matching was done by mixing small amounts of the pigments into the elastomer.

21. Insertion was done (Figure-VI). By this prosthesis we have tried to restore her esthetics, phonetics and her confidence level.

Post-insertion instructions regarding cleaning and maintenance of prosthesis were advised to the patient. The patient was recalled for a follow-up visit after a week. After one week follow-up these was marked improvement in prosthesis retention \& stability along with improved masticatory oral speech function. 


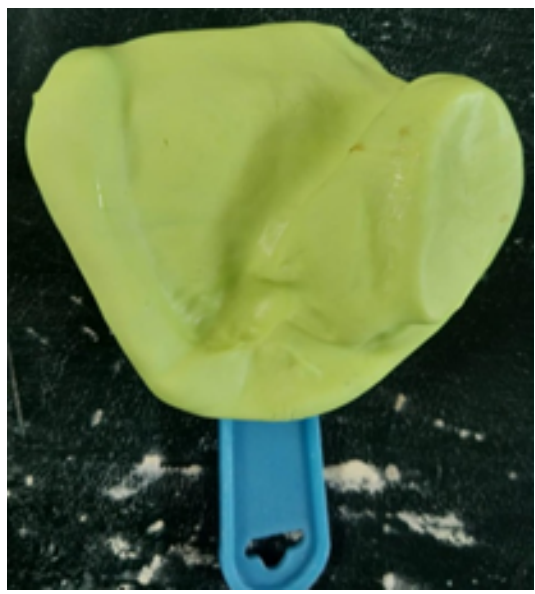

Figure-I: Putty impression

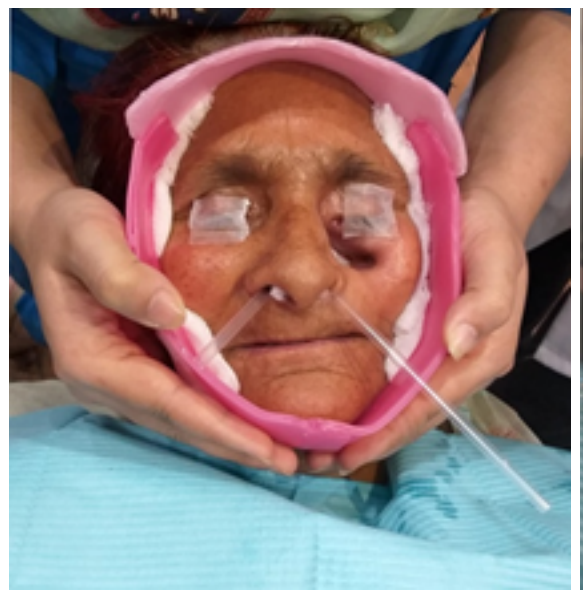

Figure-II: Placement of wax confiner

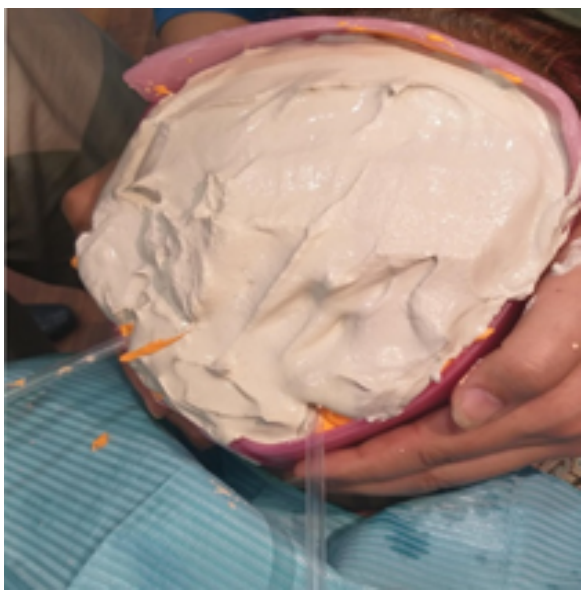

Figure-III: POP poured on alginate

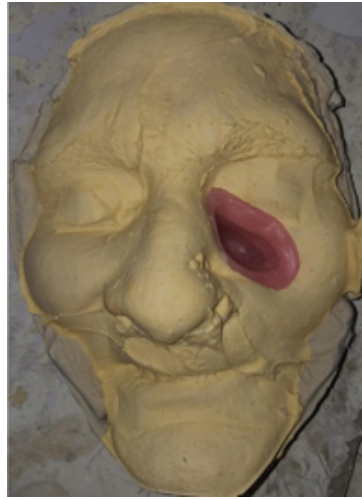

Figure-IV: Master cast view

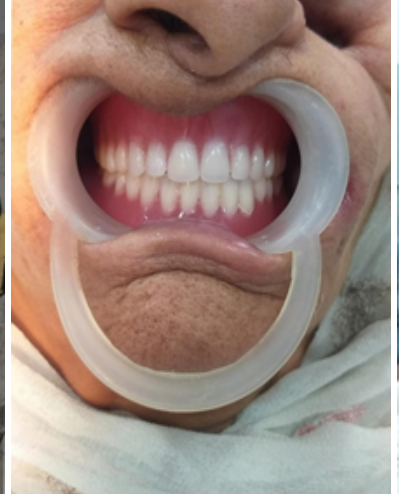

Figure-V: Insertion of dentures

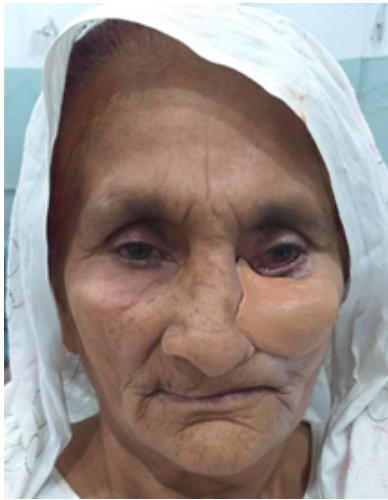

Figure-VI: (a): Post-operative

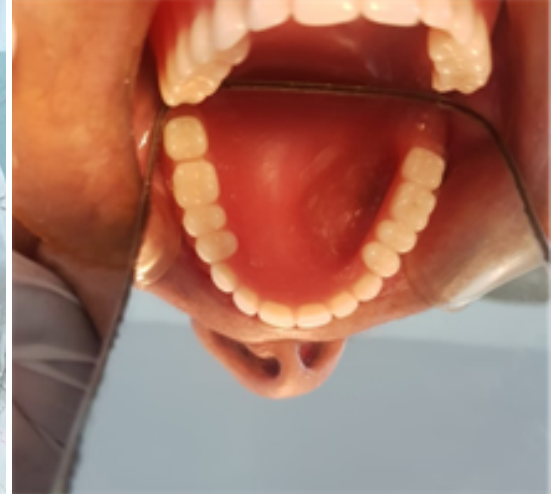

Figure-VII (b): Post-operative

\section{DISCUSSION:}

We have rehabilitated this patient with intraoral and extra-oral prosthesis retained together with magnetic attachments.

Use of magnetic stabilizers has helped in maintaining and stabilizing facial prosthesis related to oral prosthesis. This prosthesis has certain limitations as well. Like as it was made in heat cure acrylic and both intrinsic and extrinsic stains were given, extrinsic stains fade with time and need to be repaired within three months.

Factors causing color instability were accumulation of stains, dehydration, water absorption, infiltration, surface roughness, chemical degradation, degradation from use, and continuous formation of pigments ${ }^{[11]}$.

Silicone is always a better option as it records fine details and light in weight. Color staining results are also remarkable, but due to limited resources, acrylic is used as it is affordable and easily repairable.

The restoration of mastication, deglutition, phonation and esthetics was possible with the help of this prosthesis psychological improvement. It improved patient's quality of life ${ }^{[12]}$. After the prosthesis was inserted, along with follow-up interview was taken from the patient and it was noted that prosthesis aids her in chewing and in prevention of leakage of nasal 
fluids into oral cavity. Her airway was also maintained. A meaningful improvement in esthetics and function as well as psychological improvement occurred which facilitated the patient's re-integration into the society.

CONFLICT OF INTEREST: All authors disclose no conflict of interest.

\section{GRANT SUPPORT\& FINANCIAL DISCLOSURES: None.}

* Putty: Polyvinyl siloxane is an additionreaction silicone elastomer. It is a viscous liquid that sets quickly into rubber like solid taking the shape of whatever surface it was lying against while curing.

** Dental Stone: It is a calcined gypsum derivative similar to dental plaster but stronger than plaster of Paris. It is used for making dental casts and dies.

$* * *$ Separating media: Vaseline

$* * * *$ Impression material: Alginate, it is an elastic, irreversible hydrocolloid impression material. It is most frequently used dental material.

\section{REFERENCES:}

1. Narender Singh, Maj, R.K.Dhiman, Col, Dinesh Kumar Article in Med J Armed Forces India.2015 Dec published online 2014 April 26.

2. Thiele OC, Brom J, Dunsche A, et al. The current state of facial prosthetics- $A$ multicenter analysis. Journal of Craniomaxillofacial Surgery 2015 Sep 43(7): 1038-41.

3. Vallittu PK, Kokkonen M. Deflection fatigue of cobalt-chromium, titanium, and gold alloy cast denture clasp. J Prosthet Dent 1995; 74:412-9.

4. Yakir A. Arteaga in Esthetic Dentistry ( $3^{\text {rd }}$
Edition) 2015.

5. Kim, Soung Min article Magnet-Retained Orbital Prosthesis using a dental implant, Journal of Craniofacial Surgery March 2017 Volume 28 Issue 2 p e151-e152.

6. Barron JB, Rubenstein JE, Archibald D, Manor RE. Two piece orbital prosthesis. J Prosthet Dent 1983; 49:396-88.

7. Marcelo Coelho Goiato, Aldieris Alves Pesquira, Cristina Ramos da silva in Patient satisfaction with maxillofacial prosthesis, review in Journal of Plastic, Reconstructive and Aesthetic Surgery 2009 62,175-180.

8,9. James D. Anderson, George Zarb in Prosthodontic Treatment for edentulous patients. $13^{\text {th }}$ edition page 180,190 .

10. Zarb, Hobkirk in Prosthodontics Treatment for edentulous patients, 13 th edition, page 412,413 .

11. Srivastava, et al.: Intrinsic and Extrinsic Stains and Color Bleeding in Maxillofacial Prosthesis, Heal Talk Volume 10, Issue 5, May-June 2018.

12. Nadeau J. Maxillofacial prosthesis with magnetic stabilizers. The Journal of Prosthetic Dentistry. 1956 Jan 1;6(1):1149.

\section{Author's Contribution:}

Muhammad Aamir Rafique: Study Design and final approval of manuscript.

Safana Khan: Data collection and literature search.

Hira Ahmed: Over all Supervision of data collection, analysis and manuscript writing.

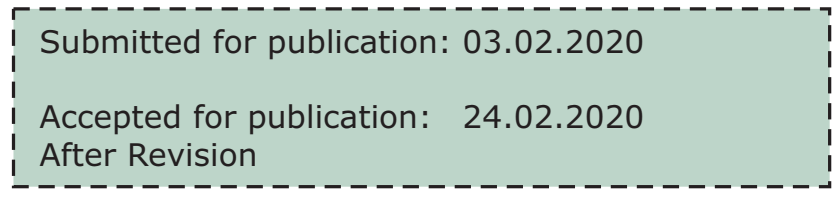

